\title{
El estudio de las prácticas políticas de los jóvenes en Internet
}

\author{
MARÍA REBECA PADILLA DE LA TORRE ${ }^{1}$ \\ DORISMILDA FLORES MÁRQUEZ 2
}

Los estudios internacionales sobre jóvenes y prácticas políticas en Internet se analizan con relación a los conceptos de audiencias y ciudadanía. Estos trabajos se han centrado en evaluar las prácticas políticas formales dirigidas a los jóvenes en Internet, y a la vez avanzan en la comprensión de la brecha digital, la apropiación del espacio y los nuevos sentidos de lo político.

PALABRAS ClAVE: Internet, jóvenes, cultura política, audiencias, ciudadanía.
Research on the online political practices of young people is closely linked to the concepts of audiences and citizenship. The body of literature has focused on formal political practices targeting young internet users, and contributes to clarify our understanding of digital divides, the reclaiming of space and novel conceptions of that which is "political".

KEY WORDS: Internet, young people, political culture, audiences, citizenship.

1 Universidad Autónoma de Aguascalientes, México.

Correo electrónico: mpadilla@correo.uaa.mx

2 Universidad Autónoma de Aguascalientes, México.

Correo electrónico: dorixfm@gmail.com 
La innovación que significó Internet dio lugar a varias preguntas sobre cuál sería su papel en la reconfiguración de distintos escenarios, como sucedió en su momento con las innovaciones mediáticas precedentes. En este texto, nos situamos en el escenario de lo político con relación a los jóvenes, con el objetivo de presentar un estado de la cuestión de investigaciones que abordaran el cruce entre Internet, jóvenes y política. Este trabajo se basó en una revisión de los bancos de datos internacionales de Sage y Ebsco. Los criterios que dirigieron la selección de los trabajos analizados fueron: primeramente, el identificar estudios empíricos que se hayan realizado a partir de 2000; otra situación fue que los sujetos de estudio fueran jóvenes y, finalmente, que la investigación se centrara en las prácticas que la juventud realiza en Internet, con relación al tema de la política de manera formal, de acuerdo a la ciencia política (Beck, 1997) o comprendiendo a la política como un terreno que se expande al vincularse con los asuntos públicos (Bakardjieva, 2010).

Martín-Barbero (2006) define como "un regalo con el cual insertarse en la velocidad de los tiempos" (p. 293) a las competencias que tienen la mayoría de los jóvenes para incorporarse en las nuevas lógicas y lenguajes. Esta situación plantea la siguiente pregunta: ¿qué hacen los jóvenes con este regalo o don en términos de canalizarlo para fortalecer su papel como ciudadanos? O por el contrario, ¿se diluye su potencial en prácticas relacionadas con lo lúdico, el entretenimiento o el consumo? La revisión de estudios precedentes es invaluable para identificar tendencias internacionales y para emprender la tarea de investigar qué sucede al respecto en nuestro país y localidad. ${ }^{3}$

3 Este texto se desprende de una investigación, actualmente en desarrollo: "Ciudadanía política en la red. Análisis sociocultural del uso de la Internet en prácticas políticas entre jóvenes universitarios". El objetivo es identificar y analizar las prácticas políticas que realizan los jóvenes universitarios, utilizando como soporte mediático Internet, con relación a la instancias políticas formales. La metodología consistió en trabajar con un grupo de jóvenes universitarios que llevaron diarios durante cuatro meses sobre sus actividades en Internet y además participaron en entrevistas colectivas sobre Internet y política. La recolección de datos se realizó durante la campa- 
En México se ha dado un crecimiento considerable en el acceso a Internet, el dato más antiguo en este sentido, registró 1 millón 222 mil usuarios, en 1998 (Instituto Nacional de Estadística, Geografía e Informática [INEGI], 2008); para 2009, la misma institución ha reportado 27’206,174 internautas (INEGI, 2009). Las encuestas sobre Internet señalan a los jóvenes como sus principales usuarios. Los porcentajes de penetración más elevados se encuentran entre las edades de los 12 y 19 años, con $68 \%$ y de los 20 a los 24 años con $61 \%$ (Asociación Mexicana de Internet [AMIPCI], 2010). A pesar de que en todas las edades se observa un incremento en la penetración de Internet, los jóvenes siguen siendo el grupo de edad que, con mayor énfasis, se ha apropiado de este soporte tecnológico. Por ello se han vuelto, junto con los niños, en los principales sujetos de estudio de interés para la investigación e incluso para organismos internacionales, como la Organización de las Naciones Unidas para la Educación, la Ciencia y la Cultura (UNESCO). Las razones se sitúan más allá de que constituyen el grupo más numeroso de usuarios; como ha señalado Martín-Barbero (2008), el futuro no está delante, sino que vive entre la sociedad gestando nuevos regímenes de historicidad. "Comprender las modalidades etno/sociales de esa experiencia constituye el reto de fondo que la juventud plantea a la investigación" (p. 28). Precisamente indagar cómo se lleva a cabo la apropiación de Internet entre los jóvenes, no sólo contabilizando cuántos lo hacen, sino cómo y con qué sentidos, permitirá entender la gestación de los cambios en distintos aspectos de la vida social, que en este estudio, de manera más específica, situamos en la esfera de lo político.

\section{CAMBIOS EN LAS CONCEPTUALIZACIONES}

SOBRE LAS AUDIENCIAS Y LA CIUDADANÍA

EN LA ERA DE INTERNET

La descripción y el análisis de los estudios internacionales más recientes de los jóvenes e Internet, en el marco de las prácticas políticas contemporáneas, lo emprendimos considerando dos desplazamientos centrales

ña a la gobernatura del estado, alcadía y diputados locales en la ciudad de Aguascalientes de 2010 (Padilla \& Flores, 2010). 
sobre las audiencias y la ciudadanía. Estos cambios conceptuales constituyen un marco de referencia crítico que permite entender este objeto de estudio de manera enraizada en la historia de las ideas sobre la interacción de los distintos actores sociales con los medios y ahora Internet.

Los estudios de medios y, de manera más específica, los estudios de audiencias y de recepción, recibieron con varias interrogantes la nueva presencia que significó Internet y las prácticas que detonó. Estas discusiones cobran especial relevancia al integrar un estado de la cuestión, porque nos permite situar el marco de la genealogía de las perspectivas teóricas y conceptuales que posicionan este tema de estudio. En las investigaciones precedentes sobre comunicación masiva, durante varias décadas la televisión fue el centro y el medio más estudiado y, en el caso de los estudios de recepción, la audiencia fue el concepto central.

Los cambios que introdujo Internet no sólo como el soporte principal de las nuevas tecnologías, sino además su injerencia al replantear el contexto mediático en general, se tradujeron en la posibilidad de dos posturas dicotómicas frente a la red. Livingstone (2004) explica que por una parte existía la posibilidad de negar la pertinencia de la continuidad histórica del conocimiento en torno a la comunicación mediada, replanteando de manera fundamental los estudios de comunicación masiva, o por el contrario, se podía dar el no considerar los cambios provocados por los llamados nuevos medios, argumentando que prevalecían los elementos y las lógicas esenciales, pero envueltas en nuevas formas que no deberían precipitar reconceptualizaciones radicales.

Consideramos que la pregunta que formula Livingstone (2004) ante esta dicotomía es fundamental: “¿Qué debe hacer quien investiga audiencias en la era de los nuevos medios, convergentes e interactivos?" (p.78). Coincidimos con la autora en su respuesta al reflexionar sobre la necesidad de mantener presente el conocimiento que los estudios de medios y, en particular, de la televisión, han logrado en las pasadas cinco décadas, para situarlo con atención en el estudio de la permanente reconfiguración en el ambiente comunicativo (Padilla, 2008).

Livingstone (2004) resume con precisión los logros de los estudios de medios en tres grandes aportaciones que se traducen en retos metodológicos para nuevos estudios a emprender. En primer lugar, los estudios de recepción que, desde la perspectiva de las audiencias se apoyaron fuer- 
temente en la etnografía, ahora enfrentan un asunto más complejo debido a que la experiencia de recepción de medios se vuelve cada vez más una práctica privada e íntima, difícil de observar, ya que se realiza en espacios y en horas de difícil acceso para el investigador. Por otra parte, la relación entre el lector y el texto fue trabajada de manera muy amplia desde la semiótica, como un problema de interpretación; se estudió con relación a los distintos géneros y entre las diversas variables posibles.

Internet significó una ruptura con lo anterior. Uno de los cambios clave es que la lógica de un productor hacia varios receptores se ha transformado. Actualmente, el reto es identificar y analizar las múltiples tareas posibles en variadas plataformas mediáticas, convergentes, interactivas, móviles e incluso híbridas entre sí, tornando a los receptores a su vez en productores. Precisamente éste es uno de los cambios sustanciales en donde la comunicación masiva de "uno a varios", se ha vuelto una comunicación "uno a uno", "uno a varios", "varios a uno" e incluso de "varios a varios". Mapear estas transformaciones, considerando el concepto tradicional de audiencias es el principal reto a enfrentar. El tercer cambio metodológico se refiere a la eterna pregunta sobre los efectos, la influencia o las consecuencias implícitas en los medios. Desde los ámbitos sociales y particularmente de las políticas públicas se mantienen como relevantes, sobre todo hacia grupos considerados vulnerables como los niños y los jóvenes.

El artículo de Livingstone (2004) expone que los nuevos medios hacen necesario no desechar el conocimiento previo, sino colocarlo en un marco más complejo y ampliado, incluso con conocimientos considerados superados. Con anterioridad, se habían planteado las preguntas sobre lo que significaba la presencia en sí misma de los medios, lo cual se había considerado limitado a un determinismo tecnológico; ahora comprobamos que las reflexiones sobre cómo construir marcos teórico-conceptuales acordes a los retos que plantean los cambios en el escenario mediático, nos llevan a un pensamiento integrado, donde el sentido material y de presencia de los medios y su capacidad de generar una creciente diversidad de prácticas, es tan importante como comprender la interpretación o el sentido que otorgan distintos segmentos de la audiencia a determinados contenidos mediáticos (Padilla, 2007). Con base en lo anterior, en la era de Internet, Livingstone (2004) argu- 
menta que los cambios en la naturaleza de las audiencias y sus prácticas, en el nuevo ambiente comunicativo, se vuelve un asunto central para el análisis en los estudios de medios.

Además de pensar sobre lo que significa el concepto de audiencias, es necesario reflexionar sobre el concepto de ciudadanía en la era de Internet. Hermes (2006) afirma, coincidiendo con lo anterior, que las tecnologías de información y comunicación, las cuales en su mayoría fueron posibles a partir de Internet, han impactado las prácticas ciudadanas; esto constituye una pregunta clave que se deberá resolver. Históricamente, la ciudadanía se ha entendido con relación a la formación de la opinión pública y un elemento esencial para ello ha sido la prensa, debido a su misión de nutrir con información a los ciudadanos. Sin embargo, las prácticas periodísticas como se conocían, específicamente las noticias, han tenido transformaciones significativas por el creciente acceso a contenidos mediáticos, no necesariamente provenientes de fuentes institucionalizadas periodísticas.

La voz de los públicos con relación a los temas políticos ha estado presente en los medios a través de los sondeos o encuestas de opinión. Las audiencias se han manifiestado a través de la medición estadística de la opinión pública, las cuales no han estado exentas de polémica. La conceptualización del ciudadano - con relación al ser audiencias-remitía en el contexto de la comunicación masiva hacia el comprender cómo éstas se convertían en públicos, al formarse una opinión con respecto de distintos asuntos de interés general (Ferry, 1998). En este sentido, el concepto de ciudadano, considerando estrictamente su relación con un Estado-nación, se encuentra limitado; más aún en el nuevo contexto mediático, con sus crecientes posibilidades de interacción. La perspectiva tradicional republicana que enfatiza la integración de una comunidad política más allá del individuo - a diferencia de la tradición liberal-parece que se acerca más a los sentidos actuales de formación de comunidades transnacionales o alternativas, con base en Internet, que co-existen con las comunidades que integran los Estados-naciones.

El concepto de ciudadano individual y su relación con un Estadonación se amplía fuertemente con apoyo de los marcos de los estudios culturales si se incorpora la construcción de comunidades entre ciudadanos, a partir de diversas formaciones identitarias. La mirada desde lo 
que se entiende como "ciudadanía cultural" permite una comprensión más clara de identidades y competencias compartidas entre audiencias que conforman públicos interesados en temáticas comunes y con opiniones similares. Hermes (2006) argumenta que:

La ciudadanía cultural ofrece las reglas básicas para la interpretación y la evaluación de los espacios en donde se es capaz de motivarse, asustarse, escandalizarse o comprometerse con diversos estados de ánimo y sentimientos que se conectan con los usos populares de los medios; en lugar de considerar al ciudadano meramente informado a través del periódico (p. 303).

Las características de Internet abren las posibilidades para que los individuos se integren entre sí y conformen públicos, en el sentido de compartir identidades y marcos culturales comunes. Por estas razones, se vuelve muy pertinente la perspectiva de Hermes para situar las posibilidades y el papel de Internet en la conformación de la ciudadanía contemporánea, especialmente en el caso de los jóvenes. Los vínculos culturales, en ciertas situaciones detonantes, se vuelven conexiones entre ciudadanos preocupados y dispuestos a participar en asuntos públicos.

Lo que estamos presenciando no es sólo el encuentro de grupos de amigos, sino además -en ocasiones- de extraños que tienen como propósito conectarse con otros, con base en agendas y metas compartidas. Los eventos mediáticos clarifican que los vínculos culturales pueden ser tan fuertes como aquéllos que se forman en las arenas políticas y quizás incluso más valiosos, en el sentido que conectan problemas políticos y respuestas hacia mundos fuera del dominio de la política en sí (Hermes, 2006, p. 306).

Esta discusión plantea que nos encontramos en un cambio trascendental, no en el ciudadano, sino en las prácticas ciudadanas. Particularmente en el caso de Internet, se desplaza el concepto del ciudadano informado a través de la prensa, hacia la conformación de comunidades por parte de los mismos ciudadanos; esto se encuentra ligado de manera directa con un cambio generacional en el sentido y el uso de los medios. 


\section{Consideraciones sobre la ciudadanía y los jóvenes}

En la búsqueda de discusiones clave sobre las prácticas políticas en Internet entre jóvenes, encontramos confluencias en dos autores. Hermes (2006) apunta hacia dos situaciones esenciales en la naturaleza de los cambios: por una parte la manera en la cual la conceptualización de ciudadanía se encuentra interrogada a partir de nuevos sentidos en las prácticas políticas y, por otra, la dimensión generacional en los actores sociales que llevan a cabo estas prácticas con apoyo de Internet como una herramienta que da lugar a nuevos sentidos y prácticas en la ciudadanía política.

En el caso de América Latina, Reguillo (2003) realiza un detenido análisis de la atmósfera que sitúa a los jóvenes como sujetos históricos y concluye que "lo joven" es una categoría de la cual no deben darse por supuestas sus características, sino que se debe asumir a los jóvenes como "heterogéneos, complejos y portadores de proyectos diferenciales" (p. 2); coincide con Hermes (2006), al plantear que la conceptualización formal de ciudadanía, entendida como el mutuo contrato de derechos y obligaciones entre el Estado y el individuo, con base en elementos de pertenencia y tres dimensiones - civil, política y social-, se encuentra seriamente amenazada precisamente porque, en la realidad, la diversidad cultural impone diversos sentidos y prácticas en torno a los asuntos públicos y la política. Ambos autores abogan por incorporar la dimensión cultural, es decir la "ciudadanía cultural", como una manera de abrir el pensamiento para que sea posible comprender los sentidos y cambios que se dan desde la perspectiva de la realidad de los jóvenes:

Coloca en el debate aspectos que no fueron considerados en las otras dimensiones: la cultura como plataforma para la ciudadanía o en otras palabras, la consideración de las pertenencias y adscripciones de carácter cultural como componentes indisociables en la definición de ciudadanía (Reguillo, 2003, p.5).

Reguillo fundamenta que la comprensión de las formas emergentes de participación en lo público y en la política necesariamente tiene que entenderse desde la cultura, la culturalización de la política es un tema clave en las ciencias sociales. Esto coincide plenamente con estudios 
previos que hemos realizado entre identidades urbanas: la cultura constituye un potente eje de análisis para entender no sólo cómo se sitúan los ciudadanos, sino además sus posibilidades de agencia y de compromiso para con otros ciudadanos y en general con la comunidad política mayor como el Estado o el país (Padilla, 2009). En este sentido, Reguillo señala que la ciudadanía juvenil necesariamente debe entenderse como una "ciudadanía policéntrica", que permite comprender lo emergente y no sólo la ciudadanía política formal a través de la organización tradicional o la participación electoral. Para entender a la ciudadanía en clave de los jóvenes, es necesario plantear a la "ciudadanía cultural" como una categoría síntesis que complejice el análisis, asumiendo la diversidad y los nuevos sentidos en las prácticas políticas, anclados en las lógicas de la identidad y la cultura. Precisamente, ahora damos a conocer los estudios más recientes sobre jóvenes e Internet en el contexto de lo político.

\section{ESTUDIOS INTERNACIONALES SOBRE}

JÓVENES, POLÍTICA E INTERNET

A principios de esta década se registró en la esfera internacional la emergencia de estudios sobre Internet en relación con los niños y jóvenes. Ahora, en 2010, se trata de una línea plenamente relevante, ya que cada vez más las nuevas generaciones median varias de sus actividades a través de las tecnologías de información y comunicación. Sonia Livingstone (2003) es una investigadora clave en este tema, ella ha clasificado los trabajos de investigación en cuatro grandes categorías:

1. Comunicación, identidad y participación.

2. Educación, aprendizaje y alfabetización.

3. Los peligros de la exclusión y la brecha digital.

4. Los riesgos del uso, el contenido y la comercialización.

Estos núcleos temáticos se han abordado a partir de tres perspectivas: la primera es la sociología de la infancia y los estudios de audiencias, los cuales se centran en los niños, más que en la tecnología o los medios y los conciben como intérpretes activos en línea; en segundo 
lugar, se ubican las investigaciones sobre niños e Internet, que consideran que se debe trascender el estudio del acceso y se debe indagar más en la naturaleza de Internet; finalmente, los estudios que comprenden que los nuevos medios no remplazan a los tradicionales, sino que enriquecen y redefinen el ambiente mediático.

En 2008, la misma autora reflexionó sobre las transformaciones en la investigación sobre la participación juvenil e Internet. Ha señalado que, a diferencia de 2003, cuando había pocos estudios e iniciativas relativas a la participación juvenil usando Internet, en los años posteriores se registró una explosión de iniciativas de gobiernos, industria, organizaciones juveniles y activistas sociales buscando enganchar a esta supuesta generación políticamente apática, pero digitalmente entusiasta, capitalizando la apropiación creativa y con frecuencia experta que los jóvenes hacen de Internet. También la investigación se incrementó en la evaluación de estas iniciativas (Livingstone, 2008).

A partir del análisis de los estudios internacionales más recientes encontramos, al igual que Livingstone, que una veta de investigación que se ha consolidado se centra en evaluar la comunicación política dirigida a los jóvenes a través de Internet. Además, otros estudios se dedican a dar seguimiento a la participación política y ciudadana de los jóvenes en este medio y su vinculación con acciones políticas más tradicionales. Por otra parte, existe un cuerpo de estudios que en primera instancia no abordan de manera directa y evidente el estudio de los jóvenes y la política en Internet, pero permiten avanzar en la comprensión del interés de los jóvenes no tanto en las prácticas políticas formales, pero sí en los problemas y asuntos públicos. Dichos estudios vinculan lo público con la política formal e institucional, en primer lugar, al comprender a la brecha digital como un elemento clave de desigualdad entre los ciudadanos más jóvenes. Una segunda categoría de estos estudios explora los sentidos que otorgan los jóvenes a las prácticas en Internet, en donde revelan su capacidad de reconocer posturas comunes ante distintos problemas y organizarse para dar respuesta a ellos. Por último, otro grupo de estudios avanza en la comprensión de la producción de espacios a través de sus prácticas en Internet y su vinculación con la apropiación juvenil de espacios presenciales para configurar identidades. Presentamos enseguida de 
manera más detallada las tendencias, en estas temáticas, que identificamos en el ámbito internacional.

Análisis de la comunicación política en Internet dirigida a los jóvenes Existen estudios que se insertan en las corrientes más tradicionales de los estudios de medios, en el sentido de que su objetivo es evaluar la efectividad de la comunicación política, en este caso a través de Internet. Los portales son actualmente una manera emergente de interpelar a los ciudadanos. Olsson (2008b) analiza a los usuarios jóvenes y a los productores de sitios electrónicos con distintos propósitos, como promoción de partidos políticos, activistas y comerciales. Sus resultados muestran que la diferencias en las lógicas de producción se deben a los recursos con los cuales se cuenta, siendo central si el sitio es producido por voluntarios o profesionales y además el sentido de sus orientaciones políticas.

En el contexto de las elecciones parlamentarias europeas se investigó el papel que jugó Internet para comprometer a los jóvenes con la acción política (Ward, 2005). Se analizaron 46 distintos sitios electrónicos y la manera en la cual se dirigieron a la juventud, este trabajo se integra a un proyecto internacional que compara sitios políticos alrededor del mundo.

En el mismo sentido, se analizaron 200 páginas electrónicas de candidatos y partidos políticos en Estados Unidos, dirigidas a votantes jóvenes, durante las elecciones en los años 2002 y 2004, comparando su papel entre ambos años electorales (Xenos \& Bennet, 2007). Además del análisis de los sitios, se estudiaron las redes de relación que crean los jóvenes en línea y sus vínculos con estas páginas. Los resultados mostraron que aún cuando creció significativamente la esfera de compromiso juvenil en Internet, éste se encontró desconectado con relación a lo electoral.

En las elecciones de 2006, Xenos y Kyoung (2008) estudiaron los efectos de los portales políticos en los jóvenes universitarios en Estados Unidos. La investigación mostró que los efectos son sustanciales cuando los jóvenes se encuentran ya interesados y comprometidos políticamente.

A partir del mismo interés por los portales y los jóvenes, Livingstone (2007) estudió a través de entrevistas, cómo los jóvenes interpretaban sitios en la red creados para ellos por instituciones públicas, con fines educativos y de apoyo social. El estudio construye categorías inte- 
resantes de análisis para evaluar la promoción de la participación de los jóvenes en línea, la capacidad de alfabetización y la relaciones de poder entre productores y usuarios.

Durante las elecciones de 2008 en Estados Unidos, Wu (2009) exploró las páginas de Facebook de los candidatos presidenciales. Se preguntó si en ellas los jóvenes lograban trasladar sus sentidos de entretenimiento y funciones de conexión entre pares, hacia la facilitación del compromiso político. Particularmente, se estudió el caso de Obama y se encontró que el vínculo con el candidato se dio en términos de una fan democracy. Este concepto es trabajado por Van Zoonen (2005), para explicar cómo los jóvenes se comprometen con las figuras políticas, vinculados a las formas tradicionales de la cultura y comunicación masiva popular.

\section{La participación política y ciudadana de los jóvenes en Internet}

Otro cuerpo de estudios coloca el foco de interés en las formas de participación política y ciudadana de los jóvenes, como un objeto en constante transformación. En este sentido, Blasco y Hansen (2006), señalan con preocupación la presencia de aspiraciones cosmopolitas y una concepción de ciudadanía posnacional, en la tendencia de la educación de los jóvenes. Además, identifican un cuestionamiento de los académicos hacia las visiones normativas de lo político que señalan cómo deberían ser las elecciones y cómo deberían participar los ciudadanos, e incluso con frecuencia describen una juventud desinteresada en la ciudadanía democrática.

Coleman (2006) plantea que la ciudadanía inclusiva requiere un abordaje más imaginativo para la inserción cívica de los jóvenes, donde las políticas públicas no vayan hacia los jóvenes, sino que se parta de su perspectiva para formularlas. Harris (2008) sostiene que en el debate sobre juventud, tecnologías de información y comunicación y participación política es importante ver que los nuevos paisajes socioeconómicos han cambiado radicalmente el significado de ciudadanía, política y participación. Como ejemplo señala cómo las mujeres desarrollan nuevos modos de activismo y subjetividad política.

En el cruce entre jóvenes, participación cívica e Internet, Banaji (2008) abordó el activismo de los jóvenes en Inglaterra, mediante el análisis de sitios electrónicos y entrevistas con sus productores y usua- 
rios. En su estudio, concluyó que los jóvenes están interesados en hacer escuchar sus voces de distintas formas. Encontró que las motivaciones para la acción cívica por parte de este grupo de edad, tienen que ver con sus experiencias en la vida cotidiana, sus ideologías, sus contextos vecinales y sociales, sus encuentros políticos, así como sus lealtades y compromisos emocionales.

Wu (2009), a partir del concepto de fan democracy de Van Zoonen analizó el uso de Facebook de los partidarios de Barack Obama, en la elección presidencial de 2008 en Estados Unidos. El autor señaló que la página de Obama en Facebook siguió la lógica de la fan democracy, es decir, que los electores actúan como fans, frente a una figura política a la que entienden como una celebridad. Sus partidarios estuvieron motivados afectivamente para intercambiar información, opiniones y movilizarse entre sus pares; todas estas acciones son formas significativas de compromiso político.

El interés por promover la conciencia y participación política se presentó en una investigación vinculada al campo de la educación cívica; en donde se promueve el uso de Internet para comunicar a estudiantes entre sí, con candidatos, para participar en encuestas y, en general, propiciar el compromiso político entre los jóvenes. Éste fue implementado en Philadelphia, Estados Unidos, como parte del programa Student Voices, el cual comprobó a través de encuestas aplicadas a estudiantes al principio y al final del estudio que hubo cambios significativos al elevar el interés de los jóvenes hacia la política (Feldman, Pasek, Romer \& Jamieson, 2007). En el mismo sentido, Jang (2005) evaluó el programa Kids Voting of Western New York y evidenció que la promoción del uso de Internet para la conversación política incrementa significativamente entre los jóvenes su motivación, interés e información en torno al tema.

La mayoría de los estudios que hemos descrito tienen como objetivo analizar la promoción e integración de los jóvenes en las prácticas políticas; sin embargo un trabajo se centró en los jóvenes que ya participaban activamente. Olsson (2008a) se preguntó cuáles eran los usos que le daban a Internet quienes militaban formalmente en movimientos políticos alternativos. Entre jóvenes activistas suecos, encontró que es considerado como central en sus actividades políticas, debido a que permite la conformación de redes. El estudio reveló que es indis- 
pensable conocer las prácticas en redes porque dan lugar a formaciones sociales para entender lo político en la era digital.

\section{Los jóvenes y la brecha digital}

En varios trabajos empíricos, encontramos que en la esfera internacional, incluso en países desarrollados, existe una preocupación por analizar la brecha digital entre jóvenes, debido a que de manera creciente se vuelve una instancia más de desigualdad. Estos estudios hacen visible que los sujetos se colocan de manera diferenciada no sólo en el acceso a Internet, sino en sus competencias para aprovechar favorablemente su uso, a partir de la manera en la cual se encuentra situado, en un país, con relación a su condición racial, la edad, el género, su posición socioeconómica y sus capitales sociales, culturales y económicos. Existen varios proyectos de investigación que no sólo analizan las principales causas o consecuencias en estas diferencias, sino además ligan el estudio con proyectos encaminados a resolver la situación mediante propuestas de políticas públicas, la implementación de actividades educativas para incrementar la alfabetización mediática y la instalación de proyectos comunitarios para ofrecer no sólo el acceso, sino una formación en el uso de Internet a comunidades con escaso acceso a esta tecnología (Alvermann, 2004; Cheong, 2008; Clark, 2003; Elias \& Lemish, 2009; D’Haenens, Koeman \& Saeys, 2007; Lee, 2008; Livingstone \& Helsper, 2007; Umrani \& Ghadially, 2008; Wilska \& Pedrozo, 2007).

\section{Los sentidos que otorgan los jóvenes a las prácticas en Internet}

Paulatinamente, en los estudios internacionales se ha avanzado hacia una clasificación de las prácticas que realizan los jóvenes en Internet. A pesar de haber sido efectuados en países cuyas condiciones son muy distintas a las nuestras, este antecedente ofrece un punto de partida para construir unas primeras categorías de análisis que podrán ponerse a prueba en nuestro propio contexto. Kaare, Brandtzaeg, Heim y Endestad (2007) trabajaron con niños y adolescentes noruegos de 10 a 12 años para determinar los usos de su comunicación mediada por computadora. Estos autores construyeron seis categorías: a) mediación de información, práctica y mensajes; b) mediación de intereses y pasatiempos; 
c) búsqueda de contacto con otros; d) comunicación con fuerte contenido emocional y simbólico; e) comunicación con el sexo opuesto, y f) comunicación para intimidar y acosar.

Con un grupo de edad más elevado, jóvenes de 15 a 25 años, Tully (2003) también aporta un cuerpo de categorías de análisis para explicar los sentidos que otorgan los jóvenes a su relación con las nuevas tecnologías. Se presentó una clasificación de los sentidos que adquieren sus experiencias con la tecnología, los cuales se refieren a sentidos de progreso, de capital simbólico, de diferenciación social, como medio de experiencias y como agente de orden en su vida cotidiana.

Revisando estos estudios, identificamos claramente un cuerpo de trabajos en donde prevalece, entre los jóvenes, el utilizar los distintos recursos en Internet para crear relaciones con pares y construir comunidades afines, al identificarse como miembros de subculturas juveniles o al compartir rasgos sobre estilos de vida. Internet se vuelve un recurso valioso para lograr vínculos con quienes viven a partir de los mismos códigos culturales y competencias comunicativas, conformando redes que integran comunidades. La pertenencia y el compartir con otros iguales, tiene de manera secundaria un sentido de apoyo para enfrentar distintos problemas comunes como problemas de salud, una discapacidad o un estado anímico ante la vida como la depresión (Hwang, Cheong \& Feeley, 2009; Livingstone, 2008; Orgad, 2006; Söderström, 2009; Wilson \& Atkinson, 2005).

\section{La producción de espacios a través de las prácticas en Internet}

Varios estudios parecen confluir en la perspectiva del "giro espacial" en los estudios de medios (Falkheimer \& Jansson, 2006), debido a que sus resultados muestran que las prácticas en Internet permiten conformar espacios virtuales, alternos a los espacios presenciales en varias dimensiones de la vida cotidiana entre los jóvenes.

Hodkinson y Lincoln (2008), a partir de una exploración sobre los usos del espacio personal de los jóvenes, señalan a la habitación como el primero que asumen como propio, ya que se trata de un lugar seguro para sí mismos; se relaciona con la construcción reflexiva de la identidad personal y además facilita la interacción privada con los pares. A la vez, la habitación se configura como la base material para 
las interacciones en espacios virtuales. Estas intersecciones entre espacios físicos y virtuales se colocan como una veta pertinente para la investigación social.

Este cruce es también abordado, de algún modo, por Van Lieshout y Aarts (2008), quienes analizaron las percepciones y prácticas de jóvenes e inmigrantes, en torno a los espacios públicos en Holanda, partiendo de la idea de que estos grupos minoritarios no necesariamente están representados en la formulación de políticas públicas. Las autoras señalan la relevancia del espacio físico para el cumplimiento de funciones sociales, como la construcción de identidades, en el contexto actual caracterizado por la cada vez menor disponibilidad del espacio público y un mayor uso de los espacios virtuales.

En el estudio de la cultura popular juvenil y de los movimientos sociales emergentes, Yang (2007) realizó un trabajo etnográfico amplio en torno a las marchas de estudiantes en defensa de los derechos de los inmigrantes, en San Francisco, en marzo de 2006. Su investigación tuvo tres fases: el antes, el durante y el después de las marchas. Observó que la movilización juvenil se basa en el consenso popular alrededor de un asunto específico donde resaltan la ausencia de un liderazgo político formal y la comunicación simultánea que permiten los nuevos medios. El estudio define con claridad, por un lado, la conexión entre espacios físicos y virtuales, y por otro, las transformaciones en el espacio virtual, ya que en los meses posteriores a las marchas, el autor registró cierta politización en MySpace, una red virtual, cuyos usos no se caracterizan por lo político, sino por el entretenimiento y lo lúdico.

La discusión sobre espacios físicos y virtuales resulta cada vez más compleja y desafiante, es claro que no se trata de espacios paralelos, sino que, de manera cotidiana, se cruzan en las prácticas comunicativas que los jóvenes han naturalizado.

\section{CONCLUSIONES}

A pesar de que ya han transcurrido varios años desde la emergencia de Internet, aún no termina del todo una etapa de preguntas en torno a la red, que ya se vivió en la historia durante la emergencia de la fotografía, el cine y la televisión, los cuales fueron nuevos medios en su momento. 
Podríamos señalar que las preguntas se sintetizan en cuestionar si este nuevo medio redundará en un beneficio, o si por el contrario representará un riesgo para el ser humano. Pareciera que aún no se aprende que la aproximación en términos dicotómicos entre lo blanco y lo negro, hacia la tecnología, no conduce a resultados productivos. En el caso de Internet y lo político se derivan igualmente dos discursos contrastantes: por un lado, se afirma que su naturaleza conducirá necesariamente a un horizonte democrático, o por el contrario, se enfatizan los riesgos ante la delincuencia, el abuso, la vigilancia y el control; además de señalar que tiene un efecto negativo en las habilidades comunicativas y en la educación de los jóvenes.

El camino más pertinente es promover el estudio empírico de lo que acontece en Internet para que sea posible, como afirma MartínBarbero (2006), "cartografiar" una realidad no del todo clara y más tendiente hacia los matices que hacia lo absoluto. Particularmente, esto cobra relevancia, dado que en México hay pocos estudios que trabajen considerando este cruce entre jóvenes, política e Internet.

Los estudios de Internet se han configurado como un campo de investigación, cuyas aportaciones son fundamentales para comprender lo social, en la era de la información. Se ha registrado una evolución, desde los primeros estudios descriptivos, en los cuales se buscaba dar cuenta de una tecnología emergente, hasta las investigaciones más recientes, centradas en las prácticas comunicativas de los sujetos. En este sentido, durante años ha habido una tensión entre lo tecnocéntrico y lo sociocéntrico. Las experiencias han demostrado la necesidad de abordajes integrales que den cuenta tanto de las materialidades de la tecnología, como de las prácticas que ésta hace posible.

La necesaria complejidad e interdisciplinariedad en los estudios sobre Internet lleva implícito revisar lo que las anteriores innovaciones tecnológicas ya nos han enseñado, a través de investigaciones previas. Es pertinente reconocer que las actuales prácticas de los jóvenes no se centran de manera nítida sólo en Internet, se vuelve evidente la creciente naturaleza intermedial e intertextual en sus prácticas mediáticas. Por último, el tema de mayor relevancia que identificamos a través de estos estudios es la emergencia en la conformación de comunidades con diversos propósitos, lo cual se vuelve una práctica más accesible y fácil, a través de las mo- 
dalidades que ofrece Internet. Sin embargo, no es prudente señalar que conduce de manera automática hacia la organización y fortalecimiento de la ciudadanía. Aún falta mayor trabajo de análisis y reflexión, para identificar cuáles son las situaciones o elementos que lo hagan posible, tarea para la cual esperamos que el presente texto contribuya.

\section{Bibliografia}

Alvermann, D. E. (2004). Media, information communication technologies, and youth literacies: A cultural studies perspective. American Behavioral Scientist, 48, 78-83.

Asociación Mexicana de Internet-AMIPCI (2010). Estudio AMIPCI 2009 sobre hábitos de los usuarios de Internet en México. Recuperado el 19 de mayo de 2010 de http://www.amipci.org.mx/estudios/temp/ Estudiofinalversion1110-0198933001274287495OB.pdf

Banaji, S. (2008). The trouble with civic: A snapshot of young people's civic and political engagements in twenty-first-century democracies. Journal of Youth Studies, 11 (5), 543-560.

Bakardjieva, M. (2010). The Internet and subactivism. Cultivating young citizenship in everyday life. En T. Olsson \& P. Dahlgren (Eds.), Young people, ICTs and democracy. Theories, policies, and websites (pp. 129-146). Gotemburgo, Suecia: University of Gothenburg-Nordicom.

Beck, U. (1997). The reinvention of politics: Rethinking modernity in the global social order. Cambridge: Poltical press.

Blasco, M. \& Hansen, H. K. (2006). Cosmopolitan aspirations: New media, citizenship education and youth in Latin America. Citizenship Studies, 10 (4), 469-488.

Cheong, P. H. (2008). The young and techless? Investigating Internet use and problem-solving behaviors of young adults in Singapore. New Media \& Society, 10, 771-791.

Clark, L. S. (2003). Challenges of social good in the world of Grand Theft Auto and Barbie: A case study of a community computer center for youth. New Media \& Society, 5 (1), 95-116.

Coleman, S. (2006). Digital voices and analogue citizenship. Bridging the gap between young people and the democratic process. Public Policy Research, 13 (4), 257-261. 
Elias, N. \& Lemish, D. (2009). Spinning the web of identity: The roles of the Internet in the lives of immigrant adolescents. New Media \& Society, 11, 533-551.

D’Haenens, L., Koeman, J. \& Saeys, F. (2007). Digital citizenship among ethnic minority youths in the Netherlands and Flanders. New Media \& Society, 9 (2), 278-299.

Falkheimer, J. \& Jansson, A. (Eds.). (2006). Geographies of communication. The spatial turn in media studies. Gotemburgo, Suecia: University of Gothenburg-Nordicom.

Feldman, L., Pasek, J., Romer, D. \& Jamieson, K. H. (2007). Identifying best practices in civic education: Lessong from the student voices program. American Journal of Education, 114, 75-100.

Ferry, J. (1998). Las transformaciones de la publicidad política. En J. M. Ferry, D. Wolton, M. R. Segura et al. El nuevo espacio público (pp. 13-27). Barcelona: Gedisa.

Harris, A. (2008). Yount women, late modern politics, and the participatory possibilities of online cultures. Journal of Youth Studies, 11 (5), 481-495.

Hermes, J. (2006). Citizenship in the age of the Internet. European Journal of Communication, 21 (3), 295-309.

Hodkinson, P.1 \& Lincoln, S. (2008). Online journals as virtual bedrooms?: Young people, identity and personal space. Young, 16 (1), 27-46.

Hwang, J. M., Cheong, P. H. \& Feeley, T. H. (2009). Being young and feeling blue in Taiwan: Examining adolescent depressive mood and online and offline activities. New Media \& Society, 11 (7), 1-21.

Instituto Nacional de Estadística, Geografía e Informática-INEGI (2008).

Usuarios de Internet por países seleccionados, 1998 a 2008. Recuperado el 4 de enero de 2010 de http://www.inegi.org.mx/est/contenidos/espanol/rutinas/ept.asp?t=tinf142\&s $=$ est\&c $=4870$

Instituto Nacional de Estadística, Geografía e Informática-INEGI (2009). Usuarios de las tecnologías de información, 2001 a 2009. Recuperado el 4 de enero de 2010 de http://www.inegi.org.mx/est/ contenidos/espanol/rutinas/ept.asp?t=tinf204\&s $=$ est\&c $=5931$

Jang, W. Y. (2005). Analysis of the mediating effect of political discussion and Internet use variables on the relationship between civic 
education and discursive participation: A structural equations framework. Ponencia presentada en la 55th Annual Conference of the International Communication Association. Nueva York: ICA.

Kaare, B. H., Brandtzaeg, P. B., Heim, J. \& Endestad, T. (2007). In the borderland between family orientation and peer culture: The use of communication technologies among Norwegian teens. New Media \& Society, 9 (4), 603-624.

Lee, L. (2008). The impact of young people's Internet use on class boundaries and life trajectories. Sociology, 42 (1), 137-153.

Livingstone, S. (2003). Children's use of the Internet: Reflections on the emerging research agenda. New Media \& Society, 5 (2), 147-166.

Livingstone, S. (2004). The challenge of changing audiences. Or what is the audience researcher to do in the age of Internet? European Journal of Communication, 19 (1), 75-86.

Livingstone, S. (2007). The challenge of engaging youth online. Contrasting producers' and teenagers' interpretations of websites. European Journal of Communication, 22, 165-184.

Livingstone, S. (2008). Taking risky opportunities in youthful content creation: Teenagers' use of social networking sites for intimacy, privacy and self-expression. New Media \& Society, 10 (3), 393-411.

Livingstone, S. \& Helsper, E. (2007). Gradations in digital inclusion: Children, young people and the digital divide. New Media \& Society, 9 (4), 671-696.

Markham, A. \& Baym, N. (2009). Internet inquiry. Conversations about method. Thousand Oaks, CA:SAGE.

Martín-Barbero, J. (2006). A Latin American perspective on communication/cultural mediation. Global Media and Communication, 2 (3), 279-297.

Martín-Barbero, J. (2008) Jóvenes: desorden cultural y palimpsestos de identidad. En H. Cubides, M. Laverde \& C. Valderrama (Coords.), Viviendo a toda. Jóvenes, territorios culturales y nuevas sensibilidades (pp. 22-37). Colombia: Siglo del Hombre Editores.

Olsson, T. (2008a). The practices of Internet networking-a resource for alternative political movements. Information, Communication \& Society, 11 (5), 659-674. 
Olsson, T. (2008b). For activists, for potential voters, for consumers: Three modes of producing the civic web. Journal of Youth Studies, $11(5), 497-512$.

Orgad, S. (2006). The cultural dimensions of online communication: A study of breast cancer patients' Internet spaces. New Media \& Society, 8 (6), 877-899.

Padilla, R. (2007). Vivir con los medios, la textura mediática que envuelve la vida cotidiana. En G. Zalpa \& M. E. Patiño (Coords.), La vida cotidiana. Prácticas, lugares y momentos (pp. 57-84). Aguascalientes: UAA.

Padilla, R. (2008). Tecnologías mediáticas y la permanente reconfiguración del sensorium. Su estudio en el marco de las ciencias sociales. En R. Reguillo (Coord.), Territorios en reconfiguración. Perspectivas situadas en torno a la interdisciplinariedad. Cuadernos del DECS (pp.131-148). Tlaquepaque, Jalisco: ITESO.

Padilla, R. (2009). Perfiles socioculturales de ciudadanía. Identidades urbanas y geografías mediáticas. Estudio en cinco escenarios en la ciudad de Aguascalientes. Tesis doctoral. Tlaquepaque, Jalisco: ITESO.

Padilla, R. \& Flores, D. (2010). Ciudadanía política en la red. Análisis sociocultural en el uso de la Internet en prácticas políticas entre jóvenes universitarios. Proyecto de investigación. Aguascalientes: UAA.

Reguillo, R. (2003). Ciudadanías juveniles en América Latina. Última Década, 19, 1-20.

Söderström, S. (2009). Offline social ties and online use of computers: A study of disabled youth and their use of ICT advances. New Media \& Society, 11 (5), 709-727.

Tully, C. J. (2003). Growing up in technological worlds: How modern technologies shape the everyday lives of young people. Bulletin of Science, Technology \& Society, 23 (6), 444-456.

Umrani, F. \& Ghadially, R. (2008). Gender and decision-making in technology adoption among youth: A study of computer learners in India. Psychology \& Developing Societies, 20 (2), 209-227.

Van Lieshout, M. \& Aarts, N. (2008). Youth and immigrants' perspectives on public spaces. Space and Culture, 11 (4), 497-513. 
Van Zoonen, L. (2005). Entertaining the citizen: When politics and popular cultura converge. Lanham, Maryland, EE.UU.: Rowman \& Littlefield.

Ward, J. (2005). An opportunity for engagement in cyberspace: Political youth web sites during the 2004 European Parliament election campaign. Information Polity, 10, 233-246.

Wilska, T. \& Pedrozo, S. (2007). New technology and young people's consumer identities. A comparative study between Finland and Brazil. Young, 15 (4), 343-368.

Wilson, B. \& Atkinson, M. (2005). Rave and straightedge, the virtual and the real. Exploring online and offline experiences in Canadian youth subcultures. Youth \& Society, 36 (3), 276-311.

Wu, J. (2009). Facebook politics: An exploratory study of American youth's political engagement during the 2008 presidential election. Ponencia presentada en la 59th Annual Conference of the International Communication Association. Chicago: ICA.

Xenos, M. \& Bennett, W. L. (2007). The disconnection in online politics. The youth political web sphere and US election sites, 20022004. Information, Communication \& Society, 10 (4), 443-464.

Xenos, M. \& Kyoung, K. (2008). Rocking the vote and more: An experimental study of the impact of youth political portals. Journal of Information Technology \& Politics, 5(2), 175-189.

Yang, K. W. (2007). Organizing MySpace: Youth walkouts, pleasure, politics, and new media. Educational Foundations, 21 (1-2), 9-28. 